\title{
Modeling Landuse Impact on Runoff \& Erosion - A Review
}

\author{
${ }^{1}$ Amit Mehta, ${ }^{2}$ Dr. K.K. Singh \\ ${ }^{1}$ (Research Scholar) Department of civil Engineering/NIT Kurukshetra/India' \\ ${ }^{2}$ (Professor/Supervisor) Department of civil Engineering/NIT Kurukshetra/India'
}

\begin{abstract}
The modeling and forecasting of floods and their consequences requires a good approximation of the runoff. Due to lack of rainfall and runoff gauge stations, it has become difficult to understand hydraulic condition of a river basin. Before applying the techniques of modeling we need to obtain the information about the specific region such as typical terrain, type of soils, cropping system and management practices followed. In the present study we are going to discuss the various aspects of modeling and the tools available.
\end{abstract}

Keywords: Modeling, rainfall, runoff, hydrological data, watershed.

\section{Introduction}

Modeling means predicting the behavior of a system which takes some input $\&$ then produces output. Modeling act as a tool to convert rainfall into runoff that may help in policy making for a specific region in terms of effects on soil productivity, water quality, sedimentation and floods. The rainfall-runoff process is a complex, dynamic and non-linear process, which is affected by many and often inter-related physical factors. The influence of these factors and many of their combinations in generating runoff is an extremely complex physical process. During this process, the input in rainfall to the system in watershed goes through 'translation' in time and space due to variable source areas of the watershed contributing runoff at the outlet at different times.

A runoff model is a mathematical model describing rainfall-runoff relations of a rainfall catchment's area (drainage basin or watershed). More precisely, it produces the surface runoff hydrograph as a response to a rainfall hydrograph as input.

\section{Development Of Modeling Tools}

Water resources professionals have been working for decade to develop models to tackle watershed problems. There are number of watershed models differing in terms of approach, application, and ability to provide users with accurate simulation to the real situations. Watershed modeling efforts before 1960 were aimed mostly at quantitative representation of individual hydrologic processes such as surface runoff, infiltration, groundwater flow, and evapotranspiration, ( Singh and Woolhiser, 2002[1]; Chen 2004[2]; Crawford and Burges, 2004)[3], but a lack of data and computing capability hindered more integrated analysis (Freeze and Harlan, 1969[4]; (Chen 2004).

After the advent of computers in the 1960s development of the Stanford Watershed Model in 1966 (Crawford and Linsley, 1966)[5] initiated a new era of modelling efforts that included the parameters such as snowmelt runoff, stream-aquifer interaction, reservoir and channel flow routing, and water quality into watershed models such as Hydrologic Simulation Program FORTRAN (Johanson et al., [6] 1984; Singh and Woolhiser, 2002)[1] and HEC rainfall runoff and river hydraulics models (USACE, 1989). In order to maximize the net benefits of water allocation economic water demand curves (Harou et al., 2009)[7] were used to establish a conceptual framework for regional scale integrated water management models in late 1960s and early 1970s. Inspired by this many researchers have contributed to build hydro economic models of watershed systems by linking hydrological, hydro geological, hydraulic, and biogeochemical processes to economic principles to facilitate integrated planning and management of watersheds (Brouwer and Hofkes, 2008)[8]. Plethora of research has been carried out on hydro economic models (Heinz et al., 2007[9]; Brouwer and Hofkes, 2008; Harou et al., 2009), In 1990s and 2000s, which also included social and political aspects of watershed systems (Griffin, 1999; Korfmacher, 2001[10]; Beck et al., 2002[11]; Bagheri, 2006[12]; Madani and Marino, 2009[13]). After the development of Stanford Watershed model use of sophisticated models has raised at an overwhelming rate (Singh and Frevert, 2006[14]). Over the same period, watershed models have shifted from purely engineering/economic models to more integrated tools that are capable of planning, design, and management problems. Growing technological advances in remote sensing, satellites, and radar applications, combined with GIS techniques and data-base management systems (Singh and Woolhiser, 2002), have allowed for detailed spatial and temporal analyses of watershed systems. 


\section{Modeling System And Model Classification}

The modeling system is approached in a variety of manners. On the basis of this, models are majorly categorized as:

3.1 Event \& continuous simulation Models

3.2 Conceptual \& Hydrodynamic Models

3.3 Lumped \& distributed parameter models

3.4 Models with fitted or empirically derived parameters

\subsubsection{Event \& continuous simulation Models}

These two types of models although mentioned differently but based on a similar concept of temporal scale. If we consider the system of watershed then few parameters are clearly identified in short period of time while few properties are clearly identified in longer period of time e.g. for an individual storm event parameters such as infiltration, interception, overland \& channel flow, detention storage are more clearly reflected \& temporal scales may range from minutes to several hours.

But continuous model will reflect both events effect \& inter storm effects e.g. Ground water flow, evapotranspiration, soil moisture \& its effects on infiltration \& time interval can be daily, hourly, seasonal from the modeling approach. Initial conditions of system are either assigned by the modeler or approximated or calibrated, in case of event modeling some times past records are used to estimate the initial conditions. Field man (1979)(15) relates the antecedent conditions with a relatively simple CS Model.

Also In continuous simulated Model, Modeler gets the enough opportunities to model results comparison, with observed runoff. Also, longer calibration period will give opportunity to test model on a variety of hydrological conditions.

\subsubsection{Conceptual \& Hydrodynamic Model}

There is thin boundary b/w conceptual \& hydrodynamic model. To start with first black box model needs to be clarified. This is basic concept in modeling. Black box models means a relation between input and output more or less a statistical relation it is, it does not care to look into the actual physical process of the system involved which is taking input \& delivering output.

Conceptual models are based on a concept, like various physical processes are interrelated through a concept such as SCS-CN method \& if physics based understanding of hydrologic processes are used such as conservation of mass, conservation of energy then hydrodynamic models comes into picture. Actually there is thin differentiation $\mathrm{b} / \mathrm{w}$ two processes, but it is same.

\subsection{Lumped \& distributed Model}

Now, in short when hydrologic parameters varies with space ( It is accounted for by this type of modeling). Lumping means averaging parameters such as total rainfall soil characteristics, overland flow conditions with space. We ignore actual variation of these parameters with space within the flow route.

While distributed modeling gives hydrologic parameters variation based on geological variation of parameters across the watershed. Good distributed model even accounts for grid scale of watershed. Sometimes some lumping is allowed within distributed model, which gives rise to semi-distributed model. This modeling concept depends upon available data, time \& money e.g. HRU (Hydrologic response unit) is the portion under consideration which may be combination of a particular landuse \& soil type. Now, one can model for different HRU or may expose some HRUs to same rainfall condition or may look for variation of HRU with different temperatures. Every time approach \& method will decide it is lumped, semi-distributed or distributed model .For example HEC-GEOHMS \& SWAT.

\subsection{Models with fitted or empirically derived parameters}

For any model e.g. rainfall runoff model parameters values are very important. There are three main methods to find it.

1. Through calibration

2. Empirically fixed

3. From field measurement.

1. Calibration process depends upon error $\mathrm{b} / \mathrm{w}$ estimated \& observed value \& more or less a mathematical approach based upon observed value measurements.

2. Empirically derived are developed by curve fitting or by the regression analysis e.g SCS runoff curve no. method for estimating rainfall losses on ungauged watershed.

3. Field measurement are for physically measured watershed properties such as slope, channel width, roughness, hydraulic dimensions, permeability etc. 
Modeling is both for gauged \& ungauged watershed. For ungauged modeling it is better to utilize spatial datasets of properties of terrain, landuse, geological conditions while concentrating on the complex process of surface, subsurface $\&$ their interaction so as to predict quality \& quantity of drinking water.

In General, the nature of catchment $\&$ data availability will influence the choice of modeling approach to use. Other deciding factors of consideration will be catchment size, its location, steepness \& purpose of use of model.

Distributed modeling helps to identify the parameters when distributed modeling is done for gauged watersheds \& then this idea can be utilized in an extended manner to model ungauged watershed. Shawul, A. A., Alamirew, T., and Dinka, M. O.[16] found that SWAT model can be taken as a potential tool for simulation of the hydrology of unguaged watershed in mountainous areas. Also if we know system (e.g catchment characteristics) we can compare \& correlate model of two catchments which are very similar in location, terrain, soil, area \& geology \& landcover \& this means modeling model for gauged catchment \& using it for ungauged catchment, More over this way relating conceptually the physical parameters (physics based) with spatial datasets introduces the key to understand nature more mathematically \& later to handle \& manage it effectively. Now a days availability of digital spatial datasets on terrain \& cover, geologic properties of soil has revolutionized the hydrologic modeling. Radar precipitation, DEMS, GIS has really allowed to experiment more deeply the conceptual hydrodynamic modeling in a distributed manner \& enhanced understanding in managing the quantity \& quality of water resources e.g. we can model the effect of growing crop on runoff \& water balance.

Now, with the previous statistical method of forecasting, as that was based on observed data it was not simple to transfer the model for ungauged watershed. Generally stastical approach is used for forecasting peak values only. Probably combing both above approaches may lead to much better forecasting experience.

\section{Model calibration, validation \& verification}

Application of a model passes through three steps.

1. Calibration

2. Validation

3. Verification

4.1. Calibration is the process of modifying model parameters to reduce the error between the simulated stream flow \& some portion of the observed flow record. Flexibility of model is related to calibration \& is defined as the capability of a model to calibrate for a variety of different watersheds \& flow conditions. In actual accuracy of the observed flow record also matters.

4.2. Model validation is a test of forecast of model values outside the calibration period. Model divergence is related to it $\&$ it is defined as the relative accuracy of the model between the calibrated period $\&$ the validated period.

4.3. Model verification as defined by, Dendrou (1982)[17], investigates the range of conditions over which the model will producer acceptable results. Model exploration means hydrologic conditions outside of the range of a model used for calibration \& validation.

Calibration can be done manually or by Automatic (Some software provide this). Manual calibration has one benefit that modeler can reduce the errors the impact of parameter on output. It saves also some times from unrealistic output given by automatic calibration. Gan \& Burgrs (1990)[18] indicated that a good initial estimate of the model parameters is a significant factor in reducing the overall calibration effort t\& in developing a reliable set of parameters.

Divergence in model is created when the error $\mathrm{b} / \mathrm{w}$ forecasted flow is significantly greater than the model error for calibrated conditions. Tohini \& wallis (1977)[19] state that " a good fit to the calibration period may comfort the individual hydrologic model builder but it does not guarantee a model with minimum forecast divergence, and it imparts no substantial message to those concerned with comparative model quality."

Most important aspect of Modeling a system is model extrapolation that occurs when a model is simulate outside its range. e.g. simulating sediment yield in ungauged watersheds, evaluation effects of land cover changes or effect of extreme stream.

In these case observed stream flow records are not available to validate model. It generally becomes \& remains on area for researcher to explore the model validation on different range or area.

According to Lorsen (1973) [20] \& Gan \& Burges (1990) the physical basis of the model processes and physical interpretation of the model parameter provide the best indication of a models ability to extrapolate to different hydrologic conditions

V. Use of Various Models in hydrological modeling [21]

\begin{tabular}{|l|l|l|l|l|l|l|l|l|l|}
\hline Model & $\begin{array}{l}\text { Suited } \\
\text { Applicatio } \\
\text { ns }\end{array}$ & $\begin{array}{l}\text { Main } \\
\text { Components }\end{array}$ & $\begin{array}{l}\text { Runoff } \\
\text { on } \\
\text { Overla }\end{array}$ & $\begin{array}{l}\text { Subsurfa } \\
\text { ce } \\
\text { Flow }\end{array}$ & $\begin{array}{l}\text { Chemical } \\
\text { Simulation }\end{array}$ & $\begin{array}{l}\text { Spati } \\
\text { al } \\
\text { Scale }\end{array}$ & $\begin{array}{l}\text { Tempor } \\
\text { al } \\
\text { Scale }\end{array}$ & $\begin{array}{l}\text { Watershed } \\
\text { Representat } \\
\text { ion }\end{array}$ & $\begin{array}{l}\text { Availabil } \\
\text { ity }\end{array}$ \\
\hline
\end{tabular}


Modeling Landuse Impact on Runoff \& Erosion - A Review

\begin{tabular}{|c|c|c|c|c|c|c|c|c|c|}
\hline & & & nd & & & & & & \\
\hline ArcSWAT & $\begin{array}{l}\text { Suited for } \\
\text { agriculture } \\
\text { watersheds } \\
; \text { designed } \\
\text { for ungaged } \\
\text { watershed }\end{array}$ & $\begin{array}{l}\text { Runoff, } \\
\text { infiltration, } \\
\text { subsurface } \\
\text { drainage, soil } \\
\text { erosion, } \\
\text { interception \& } \\
\text { overland } \\
\text { sediment } \\
\text { transport } \\
\end{array}$ & $\begin{array}{l}\text { Mannin } \\
\text { g \& } \\
\text { continui } \\
\text { ty } \\
\text { Equatio } \\
\text { ns }\end{array}$ & $\begin{array}{l}\text { No } \\
\text { compone } \\
\text { nt }\end{array}$ & $\begin{array}{l}\text { No } \\
\text { component }\end{array}$ & 2 & 4 & $\begin{array}{l}\text { Square grids; } \\
\text { 1-D } \\
\text { Simulations }\end{array}$ & $\mathrm{P}_{\mathrm{b}}$ \\
\hline $\begin{array}{l}\text { AnnAGNP } \\
\text { S }\end{array}$ & $\begin{array}{l}\text { Suited for } \\
\text { agriculture } \\
\text { watersheds; } \\
\text { widely used } \\
\text { for } \\
\text { evaluating } \\
\text { a wide } \\
\text { variety of } \\
\text { conservatio } \\
\text { n practices } \\
\text { and } \\
\text { other BMPs } \\
\text { Hydrology, } \\
\text { sediment, } \\
\text { nutrients }\end{array}$ & $\begin{array}{l}\text { Hydrology, } \\
\text { sediment, } \\
\text { nutrients } \\
\text { and pesticide } \\
\text { transport, } \\
\text { DEM used to } \\
\text { generate grid } \\
\text { and stream } \\
\text { network }\end{array}$ & $\begin{array}{l}\mathrm{CN}, \\
\text { TR-55 } \\
\text { for peak } \\
\text { flow }\end{array}$ & $\begin{array}{l}\text { Darcy's } \\
\text { equation } \\
\mathrm{N}, \quad \mathrm{P}, \\
\text { pesti }\end{array}$ & $\begin{array}{l}\mathrm{N}, \quad \mathrm{P}, \\
\text { pesticides, } \\
\text { organiccarbo } \\
\text { n\& } \\
\text { nutrien } \\
\text { ts }\end{array}$ & 2 & $\begin{array}{l}\text { 3- daily } \\
\text { or } \\
\text { sub- } \\
\text { daily } \\
\text { steps }\end{array}$ & $\begin{array}{l}\text { Homogeneo } \\
\text { us } \\
\text { land areas, } \\
\text { reaches, \& } \\
\text { impoundmen } \\
\text { ts } \\
\mathrm{Pu} \\
\mathrm{GSSHA} / \mathrm{CA} \\
\mathrm{S}\end{array}$ & $\mathrm{P}_{\mathrm{b}}$ \\
\hline $\begin{array}{l}\text { GSSHA/C } \\
\text { AS } \\
\text { C2D }\end{array}$ & $\begin{array}{l}\text { Suited for } \\
\text { both } \\
\text { agriculture } \\
\text { or urban } \\
\text { watersheds; } \\
\text { diverse } \\
\text { modeling } \\
\text { capabilities } \\
\text { in a } \\
\text { variety of } \\
\text { climates } \\
\text { and } \\
\text { watersheds } \\
\text { with } \\
\text { complex } \\
\text { spatial } \\
\text { datasets } \\
\end{array}$ & $\begin{array}{l}\text { Spatially } \\
\text { varying rainfall; } \\
\text { rainfall excess } \\
\text { and 2-D flow } \\
\text { routing; soil } \\
\text { moisture, } \\
\text { channel } \\
\text { routing, upland } \\
\text { erosion, \& } \\
\text { sediment } \\
\text { transport }\end{array}$ & $\begin{array}{l}\text { 2-D } \\
\text { diffusiv } \\
\text { e } \\
\text { wave } \\
\text { equatio } \\
\text { ns }\end{array}$ & $\begin{array}{l}\text { No } \\
\text { compone } \\
\text { nt }\end{array}$ & $\begin{array}{l}\text { No } \\
\text { component }\end{array}$ & 2 & $4 ; 3$ & $\begin{array}{l}\text { 2-D square } \\
\text { overland } \\
\text { grids; } 1 \text {-D } \\
\text { channels }\end{array}$ & $\operatorname{Pr}$ \\
\hline $\begin{array}{l}\text { HEC- } \\
1 / \text { HECHM } \\
\mathrm{S}\end{array}$ & $\begin{array}{l}\text { Suited for } \\
\text { urban } \\
\text { watersheds; } \\
\text { widely used } \\
\text { for } \\
\text { modeling } \\
\text { floods and } \\
\text { impacts on } \\
\text { land } \\
\text { use changes }\end{array}$ & $\begin{array}{l}\text { Precipitation, } \\
\text { losses, } \\
\text { baseflow, runoff } \\
\text { transformation } \\
\text { \& routing }\end{array}$ & $\begin{array}{l}\mathrm{CN}, \\
\text { kinemat } \\
\text { ic } \\
\text { wave } \\
\text { equatio } \\
\text { ns }\end{array}$ & $\begin{array}{l}\text { No } \\
\text { compone } \\
\text { nt }\end{array}$ & $\begin{array}{l}\text { No } \\
\text { component }\end{array}$ & 1 & 4 & $\begin{array}{l}\text { Dendritic } \\
\text { network } \\
\text { or grid }\end{array}$ & $\mathrm{P}_{\mathrm{b}}$ \\
\hline HSPF & $\begin{array}{l}\text { Suited for } \\
\text { both } \\
\text { agriculture } \\
\text { or urban } \\
\text { watersheds; } \\
\text { diverse } \\
\text { water } \\
\text { quality and } \\
\text { sediment } \\
\text { transport at } \\
\text { any point } \\
\text { on the } \\
\text { watershed }\end{array}$ & $\begin{array}{l}\text { Runoff /water } \\
\text { quality } \\
\text { constituents, } \\
\text { simulation of } \\
\text { pervious/ } \\
\text { impervious } \\
\text { areas, stream \& } \\
\text { channels \& } \\
\text { mixed reservoirs }\end{array}$ & $\begin{array}{l}\text { Empiric } \\
\text { al } \\
\text { outflow }\end{array}$ & $\begin{array}{l}\text { Interflow } \\
\text { outflow, } \\
\text { percolatio } \\
\mathrm{n} \text {; } \\
\text { groundwa } \\
\text { ter } \\
\text { outflow }\end{array}$ & $\begin{array}{l}\text { Soil/water } \\
\text { temp., DO, } \\
\mathrm{CO} 2, \mathrm{~N}, \\
\mathrm{NH} 3, \\
\text { organic } \\
\mathrm{N} / \mathrm{P}, \\
\mathrm{N} / \mathrm{P}, \\
\text { pesticides }\end{array}$ & 1 & 3 & $\begin{array}{l}\text { Pervious } \\
\text { limpervious } \\
\text { land } \\
\text { areas, stream } \\
\text { channels, \& } \\
\text { mixed } \\
\text { reservoirs; } \\
\text { 1-D } \\
\text { simulations }\end{array}$ & $\mathrm{P}_{\mathrm{b}}$ \\
\hline $\begin{array}{l}\text { KINEROS } \\
2\end{array}$ & $\begin{array}{l}\text { Suited for } \\
\text { urban } \\
\text { environmen } \\
\text { ts } \\
\text { and } \\
\text { studying } \\
\text { impacts } \\
\text { of single } \\
\text { sever or }\end{array}$ & $\begin{array}{l}\text { Distributed } \\
\text { rainfall inputs, } \\
\text { rainfall excess, } \\
\text { overland flow, } \\
\text { channel routing, } \\
\text { sediment } \\
\text { transport, } \\
\text { interception, } \\
\text { infiltration, } \\
\end{array}$ & $\begin{array}{l}\text { Kinema } \\
\text { tic } \\
\text { wave } \\
\text { equatio } \\
\text { ns }\end{array}$ & $\begin{array}{l}\text { No } \\
\text { compone } \\
\text { nt }\end{array}$ & $\begin{array}{l}\text { No } \\
\text { component }\end{array}$ & 2 & 4 & $\begin{array}{l}\text { Cascade of } \\
\text { planes } \\
\& \text { channels; } \\
\text { 1-D } \\
\text { simulations }\end{array}$ & $\mathrm{P}_{\mathrm{b}}$ \\
\hline
\end{tabular}




\begin{tabular}{|c|c|c|c|c|c|c|c|c|c|}
\hline & $\begin{array}{l}\text { design } \\
\text { storm even; } \\
\text { Also can be } \\
\text { applied to } \\
\text { agriculture } \\
\text { watersheds. }\end{array}$ & $\begin{array}{l}\text { surface runoff \& } \\
\text { erosion }\end{array}$ & & & & & & & \\
\hline $\begin{array}{l}\text { MIKE } \\
\text { SHE }\end{array}$ & $\begin{array}{l}\text { Wide range } \\
\text { of spatial } \\
\text { and } \\
\text { temporal } \\
\text { scales; } \\
\text { modular } \\
\text { design } \\
\text { facilitates } \\
\text { integration } \\
\text { of other } \\
\text { models; } \\
\text { advanced } \\
\text { capabilities } \\
\text { for water } \\
\text { quality, } \\
\text { parameter } \\
\text { estimation } \\
\text { and water } \\
\text { budget } \\
\text { analysis }\end{array}$ & $\begin{array}{l}\text { Interception, } \\
\text { overland/ } \\
\text { channel flow, } \\
\text { unsaturated/ } \\
\text { saturated zone, } \\
\text { snowmelt; } \\
\text { aquifer/ rivers } \\
\text { exchange, } \\
\text { advection/disper } \\
\text { sion solutes, } \\
\text { of geochemical } \\
\text { processes, plant } \\
\text { growth, soil \& } \\
\text { erosion } \\
\text { irrigation }\end{array}$ & $\begin{array}{l}\text { 2-D } \\
\text { diffusiv } \\
\mathrm{e} \\
\text { wave } \\
\text { equatio } \\
\text { ns }\end{array}$ & $\begin{array}{l}\text { 3-D } \\
\text { groundwa } \\
\text { ter } \\
\text { flow }\end{array}$ & $\begin{array}{l}\text { Dissolved } \\
\text { conservative } \\
\text { solutes } \\
\text { in surface, } \\
\text { soil, \& } \\
\text { ground } \\
\text { waters }\end{array}$ & 2 & $\begin{array}{l}4 ; 3 ; \\
\text { variable } \\
\text { steps }\end{array}$ & $\begin{array}{l}\text { 2-D } \\
\text { rectangular } \\
\text { /square } \\
\text { overland } \\
\text { grids; 1-D } \\
\text { channels; } \\
\text { 1-D } \\
\text { unsaturated/ } \\
\text { 3-D } \\
\text { saturated } \\
\text { Flow }\end{array}$ & $\overline{P_{r}}$ \\
\hline SWAT & $\begin{array}{l}\text { Best suited } \\
\text { for } \\
\text { agriculture } \\
\text { watersheds; } \\
\text { excellent } \\
\text { for } \\
\text { calculating } \\
\text { TMDLs } \\
\text { and } \\
\text { simulating } \\
\text { a wide } \\
\text { variety of } \\
\text { conservatio } \\
\text { n practices } \\
\text { and } \\
\text { other } \\
\text { BMPs; } \\
\text { successfull } \\
\text { y } \\
\text { applied } \\
\text { across } \\
\text { watersheds } \\
\text { in } \\
\text { several } \\
\text { countries }\end{array}$ & $\begin{array}{l}\text { Hydrology, } \\
\text { weather, } \\
\text { sedimentation, } \\
\text { soil temperature } \\
\text { and } \\
\text { properties, crop } \\
\text { growth, } \\
\text { nutrients, } \\
\text { pesticides, } \\
\text { agricultural } \\
\text { management } \\
\text { and channel } \\
\text { \& reservoir } \\
\text { routing }\end{array}$ & $\begin{array}{l}\text { CN for } \\
\text { runoff ; } \\
\text { SCS } \\
\text { TR-55 } \\
\text { for } \\
\text { peak } \\
\text { flow }\end{array}$ & $\begin{array}{l}\text { Lateral } \\
\text { subsurfac } \\
\mathrm{e} \\
\text { flow/ } \\
\text { ground } \\
\text { flow }\end{array}$ & $\begin{array}{l}\mathrm{N}, \mathrm{P}, \\
\text { pesticides, } \\
\mathrm{C}\end{array}$ & 1 & $\begin{array}{l}\text { 3; daily } \\
\text { steps }\end{array}$ & $\begin{array}{l}\text { Sub-basins } \\
\text { based } \\
\text { on climate, } \\
\text { HRU, } \\
\text { ponds, } \\
\text { groundwater, } \\
\text { \& main } \\
\text { channel }\end{array}$ & $\overline{\mathrm{P}_{\mathrm{b}}}$ \\
\hline $\begin{array}{l}\text { PRMS/M } \\
\text { MS }\end{array}$ & $\begin{array}{l}\text { Suited for } \\
\text { agriculture } \\
\text { watershed; } \\
\text { modular } \\
\text { design } \\
\text { facilitates } \\
\text { integration } \\
\text { of other } \\
\text { models } \\
\text { (e.g., } \\
\text { climate } \\
\text { models) }\end{array}$ & $\begin{array}{l}\text { Hydrology and } \\
\text { surface runoff, } \\
\text { channel flow, } \\
\text { channel } \\
\text { reservoir } \\
\text { flow, soil } \\
\text { erosion, } \\
\text { overland } \\
\text { \& sediment } \\
\text { transport }\end{array}$ & $\begin{array}{l}\text { Kinema } \\
\text { tic } \\
\text { wave } \\
\text { equatio } \\
\text { ns }\end{array}$ & $\begin{array}{l}\text { No } \\
\text { compone } \\
\text { nt }\end{array}$ & $\begin{array}{l}\text { No } \\
\text { component }\end{array}$ & 2 & 4 & $\begin{array}{l}\text { Flow planes, } \\
\text { channel } \\
\text { segments, } \\
\& \text { channel } \\
\text { reservoirs; } \\
\text { 1-D } \\
\text { simulations }\end{array}$ & $\overline{\mathrm{P}_{\mathrm{b}}}$ \\
\hline $\begin{array}{l}\text { WATFLO } \\
\text { OD }\end{array}$ & $\begin{array}{l}\text { Best suited } \\
\text { for } \\
\text { agriculture } \\
\text { watershed } \\
\text { and } \\
\text { analyzing } \\
\text { hydrologic } \\
\text { and soil } \\
\text { erosion } \\
\text { on small } \\
\text { watersheds }\end{array}$ & $\begin{array}{l}\text { Weather } \\
\text { generation, } \\
\text { frozen } \\
\text { soils, snow } \\
\text { accumulation } \\
\text { and } \\
\text { melt, irrigation, } \\
\text { infiltration, } \\
\text { overland flow } \\
\text { hydraulics, } \\
\text { water balance, }\end{array}$ & $\begin{array}{l}\text { Kinema } \\
\text { tic } \\
\text { wave } \\
\text { equatio } \\
\text { ns }\end{array}$ & $\begin{array}{l}\text { Green- } \\
\text { Ampt } \\
\text { equation }\end{array}$ & $\begin{array}{l}\text { No } \\
\text { component }\end{array}$ & 2 & 3 & $\begin{array}{l}\text { Channel } \\
\text { segments } \\
\& \\
\text { impoundmen } \\
\text { ts }\end{array}$ & $\overline{\mathrm{P}_{\mathrm{b}}}$ \\
\hline
\end{tabular}




\begin{tabular}{|c|c|c|c|c|c|c|c|c|c|}
\hline & & $\begin{array}{l}\text { plant growth, } \\
\text { erosion, } \\
\text { deposition \& } \\
\text { residue } \\
\text { decomposition }\end{array}$ & & & & & & & \\
\hline $\begin{array}{l}\text { Oak } \\
\text { Ridges } \\
\text { Moraine } \\
\text { (ORM) }\end{array}$ & $\begin{array}{l}\text { Best suited } \\
\text { for glacier } \\
\text { deposits } \\
\text { including } \\
\text { tills and } \\
\text { moraine } \\
\text { deposits } \\
\text { that form } \\
\text { an east- } \\
\text { west ridge } \\
\text { ranging } \\
\text { about } 200 \\
\text { km long } \\
\text { and up to } \\
30 \mathrm{~km} \text { wide }\end{array}$ & $\begin{array}{l}\text { frozen } \\
\text { soils, snow } \\
\text { accumulation } \\
\text { and } \\
\text { melt, irrigation, } \\
\text { infiltration, } \\
\text { overland flow } \\
\text { hydraulics, } \\
\text { which forms the } \\
\text { modest smooth } \\
\text { landscape. }\end{array}$ & $\begin{array}{l}\text { Kinema } \\
\text { tic } \\
\text { wave } \\
\text { equatio } \\
\text { ns }\end{array}$ & $\begin{array}{l}\text { No } \\
\text { compone } \\
\text { nt }\end{array}$ & $\begin{array}{l}\text { No } \\
\text { component }\end{array}$ & 2 & 3 & $\begin{array}{l}\text { Channel } \\
\text { segments } \\
\& \\
\text { impoundmen } \\
\text { ts }\end{array}$ & $\mathrm{P}_{\mathrm{b}}$ \\
\hline HECRAS & $\begin{array}{l}\text { streamflow, } \\
\text { rainfall and } \\
\text { temperature } \\
\text { time series. }\end{array}$ & $\begin{array}{l}\text { Distributed } \\
\text { rainfall inputs, } \\
\text { rainfall excess, } \\
\text { overland flow, } \\
\text { channel routing, } \\
\text { sediment } \\
\text { transport, } \\
\text { interception, } \\
\text { infiltration, } \\
\text { surface runoff \& } \\
\text { erosion }\end{array}$ & $\begin{array}{l}\text { Kinema } \\
\text { tic } \\
\text { wave } \\
\text { equatio } \\
\text { ns }\end{array}$ & $\begin{array}{l}\text { No } \\
\text { compone } \\
\text { nt }\end{array}$ & $\begin{array}{l}\text { No } \\
\text { component }\end{array}$ & 2 & 4 & $\begin{array}{l}\text { Cascade of } \\
\text { planes } \\
\& \text { channels; } \\
\text { 1-D } \\
\text { simulations }\end{array}$ & $\mathrm{P}_{\mathrm{b}}$ \\
\hline SCS CN & $\begin{array}{l}\text { Suited for } \\
\text { agriculture } \\
\text { watershed; } \\
\text { modular } \\
\text { design } \\
\text { facilitates } \\
\text { integration } \\
\text { of other } \\
\text { models } \\
\text { (e.g., } \\
\text { climate } \\
\text { models) } \\
\end{array}$ & $\begin{array}{l}\text { Hydrology and } \\
\text { surface runoff, } \\
\text { channel flow, } \\
\text { channel } \\
\text { reservoir } \\
\text { flow, soil } \\
\text { erosion, } \\
\text { overland } \\
\& \text { sediment } \\
\text { transport }\end{array}$ & $\begin{array}{l}\text { CN for } \\
\text { runoff } \\
\text { SCSs }\end{array}$ & $\begin{array}{l}\text { No } \\
\text { compone } \\
\text { nt }\end{array}$ & $\begin{array}{l}\text { No } \\
\text { component }\end{array}$ & 2 & 3 & $\begin{array}{l}\text { Flow planes, } \\
\text { channel } \\
\text { segments, } \\
\& \text { channel } \\
\text { reservoirs; } \\
\text { 1-D } \\
\text { simulations }\end{array}$ & $\mathrm{P}_{\mathrm{b}}$ \\
\hline $\begin{array}{l}\text { VIC } \\
\text { (variable } \\
\text { in"ltration } \\
\text { capacity) }\end{array}$ & $\begin{array}{l}\text { Suited for } \\
\text { large river } \\
\text { basins and } \\
\text { agriculture } \\
\text { watershed; }\end{array}$ & $\begin{array}{l}\text { water yield and } \\
\text { stream f!ow are } \\
\text { the main } \\
\text { variables of } \\
\text { interest. }\end{array}$ & $\begin{array}{l}\text { Kinema } \\
\text { tic } \\
\text { wave } \\
\text { equatio } \\
\text { ns }\end{array}$ & $\begin{array}{l}\text { No } \\
\text { compone } \\
\text { nt }\end{array}$ & $\begin{array}{l}\text { No } \\
\text { component }\end{array}$ & 2 & 4 & $\begin{array}{l}\text { Cascade of } \\
\text { planes } \\
\& \text { channels; } \\
\text { 1-D } \\
\text { simulations }\end{array}$ & $\mathrm{P}_{\mathrm{b}}$ \\
\hline $\begin{array}{l}\text { InVEST } \\
2.1\end{array}$ & $\begin{array}{l}\text { local to } \\
\text { internationa } \\
1 \\
\text { conservatio } \\
\text { n } \\
\text { organizatio } \\
\text { ns, } \\
\text { government } \\
\text { agencies } \\
\text { and } \\
\text { businesses: } \\
\text { anyone an } \\
\text { with an } \\
\text { interest in } \\
\text { environmen } \\
\text { tal } \\
\text { manageme } \\
\text { nt, }\end{array}$ & \begin{tabular}{l}
\multicolumn{2}{l}{ under different } \\
land use \\
scenarios
\end{tabular} & $\begin{array}{l}\text { Kinema } \\
\text { tic } \\
\text { wave } \\
\text { equatio } \\
\text { ns }\end{array}$ & $\begin{array}{l}\text { No } \\
\text { compone } \\
\text { nt }\end{array}$ & $\begin{array}{l}\text { No } \\
\text { component }\end{array}$ & 2 & 4 & $\begin{array}{l}\text { Cascade of } \\
\text { planes } \\
\& \text { channels; } \\
\text { 1-D } \\
\text { simulations }\end{array}$ & $\overline{\mathrm{P}_{\mathrm{b}}}$ \\
\hline AGNPS & $\begin{array}{l}\text { Suited for } \\
\text { agriculture } \\
\text { watersheds }\end{array}$ & $\begin{array}{l}\text { Runoff, } \\
\text { infiltration \& } \\
\text { soil } \\
\text { erosion/sedimen } \\
\mathrm{t} \\
\text { transport }\end{array}$ & $\begin{array}{l}\text { CN, } \\
\text { TR-55 } \\
\text { for peak } \\
\text { flow }\end{array}$ & $\begin{array}{l}\text { No } \\
\text { compone } \\
\text { nt }\end{array}$ & $\begin{array}{l}\text { No } \\
\text { component }\end{array}$ & 2 & 4 & $\begin{array}{l}\text { Homogeneo } \\
\text { us } \\
\text { land areas }\end{array}$ & $\mathrm{P}_{\mathrm{b}}$ \\
\hline
\end{tabular}




\begin{tabular}{|c|c|c|c|c|c|c|c|c|c|}
\hline AIRES & $\begin{array}{l}\text { requires } \\
\text { much less } \\
\text { data and } \\
\text { expertise } \\
\text { than } \\
\text { InVEST, } \\
\text { and once } \\
\text { veri- } \\
\text { "ed, we } \\
\text { would } \\
\text { recommend } \\
\text { AIRES for } \\
\text { policy } \\
\text { makers and } \\
\text { other } \\
\text { nontechnic } \\
\text { al } \\
\text { users, or in } \\
\text { situations } \\
\text { with very } \\
\text { limited } \\
\text { data. }\end{array}$ & \begin{tabular}{l}
\multicolumn{2}{l}{ under different } \\
land use \\
scenarios
\end{tabular} & $\begin{array}{l}\text { Kinema } \\
\text { tic } \\
\text { wave } \\
\text { equatio } \\
\text { ns }\end{array}$ & $\begin{array}{l}\text { No } \\
\text { compone } \\
\text { nt }\end{array}$ & $\begin{array}{l}\text { No } \\
\text { component }\end{array}$ & 2 & 4 & $\begin{array}{l}\text { Cascade of } \\
\text { planes } \\
\& \text { channels; } \\
\text { 1-D } \\
\text { simulations }\end{array}$ & $\mathrm{P}_{\mathrm{b}}$ \\
\hline $\begin{array}{l}\text { ANSWER } \\
\text { S- } \\
2000\end{array}$ & $\begin{array}{l}\text { Suited for } \\
\text { medium } \\
\text { size } \\
\text { agriculture } \\
\text { watersheds } \\
\text {; } \\
\text { designed } \\
\text { for ungaged } \\
\text { watershed; } \\
\text { useful in } \\
\text { evaluating } \\
\text { the } \\
\text { effectivene } \\
\text { ss of } \\
\text { BMPs; } \\
\text { capable of } \\
\text { simulating } \\
\text { transformat } \\
\text { ion } \\
\text { and } \\
\text { interactions } \\
\text { between } \\
\text { four } \\
\text { nitrogen } \\
\text { pools }\end{array}$ & $\begin{array}{l}\text { Runoff; } \\
\text { infiltration, } \\
\text { water/ } \\
\text { river routing, } \\
\text { drainage, } \\
\text { river routing, } \\
\text { chemical/ } \\
\text { nutrient } \\
\text { transport }\end{array}$ & $\begin{array}{l}\text { Mannin } \\
\mathrm{g} \\
\text { equatio } \\
\mathrm{n}\end{array}$ & $\begin{array}{l}\text { Darcy's } \\
\text { equation }\end{array}$ & $\begin{array}{l}\mathrm{N}, \mathrm{P}, \\
\text { sediment } \\
\text { transport }\end{array}$ & 2 & 3 & Grid/cells & $\mathrm{P}_{b}$ \\
\hline
\end{tabular}

Type: F; Agriculture Watershed - $\mathrm{A}_{\mathrm{w}}$; Urban Watershed - $\mathrm{U}_{\mathrm{w}}$ Spatial Scale: Semi-Distributed - 1; Distributed 2

Temporal Scale: Continuous - 3; Event-base - 4 Availability: Public - $\mathrm{P}_{\mathrm{b}}$; Proprietary - $\mathrm{P}_{\mathrm{r}}$

\section{Evaluation \& selection of models}

It depends on the following critierion.

1. Ease of model use.

2. Model accuracy

3. Consistency of parameters

4. Sensitivity of output to changes in parameters

5. Theoretical limitations of the model.

6. Data limitation

Darren L. Ficklin, Yuzhou Luo and Minghua Zhang [22] concluded that SWAT simulations are able to capture the uncertainties that exists, such as model simplification, observed data errors and lack of agricultural management data.

\section{Conclusion}

As reviewed there are number of modeling tools available \& all are dependent on data availability, utility \& have their own merits and demerits. Distributed models are preferred when long term policy making decisions are in process as these gives insight to actual process involved, although conceptual lumped models 
gives ease of result calculation. Franchini \& Pasciani (1991)[23] express that for complex models, "the handling of many parameters which affect the behavior of these exchanges therefore remains without control \& difficult to check, which greatly increases the number of attempts that must be made before acceptable results can be achieved." They indicate that simpler models requires less time to achieve good results .. Generally in the light of review of models used \& discussions (woolshiser \& bracken sick, 1982[24]; james \& byrgers, 1982b[25]; Anderson and Burt 1985[26] number of criterion popped up such as 1. Model data availability 2.Model accuracy 3. Ease of model use 4. Sensitivity of model or lineages of various parameters involved. 5. Stability or spatial \& temporal vacation of parameters involved. 6.Theoretical limitations \& practical aspect of applying it. Yet modeling is experimenting new tools \& models in search of a more generalized approach model.

It can be concluded that in general distributed model gives a wide approach to take care of ecosystem, flora \& fauna \& to detect human beings impact on nature. Computers \& satellites have added further to allow simulation of actual field conditions in the lab \& make researchers more comfortable in experimenting with different distributed models.

\section{References}

[1]. Singh, V. P. \& Woolhiser, D. A. (2002). Mathematical modeling of watershed hydrology. J. Hydrol. Eng., 7(4), 270-292

[2]. Chen, C. W., Dean, J. D., Gherini, S. A. \& Goldstein, R. A. (1982). Acid rain model hydrologic module. J. Environ. Eng. ASCE 108 (E3), 455-472.

[3]. Crawford, N. H. \& Burges, S. J. (2004). History of the Stanford Watershed Model. Water Resour. Impact, 6, 3-5.

[4]. Freeze, R. A. \& Harlan, R. L. (1969). Blueprint for a physically-based, digitally-simulated hydrologic response model. J. Hydrol., 9, 237-258.

[5]. Crawford, N. H. \& Linsley, R. K. (1966). Digital simulation in hydrology: Stanford Watershed Model IV. Technical report No. 39, Department of Civil Engineering,Stanford University, 210.

[6]. Johanson, R. C., Imhoff, J. C., Kittle, J. L. \& Donigian, A. S. (1984). Hydrologic Simulation Program - FORTRAN (HSPF). User's Manual for Release 8. Report No.EPA-600/3- 84- 066.Athens, Ga.: U.S. EPA Environmental Research Lab.

[7]. Harou, J. J., Pulido-Velazquez, M. A., Rosenberg, D. E., Medellin-Azuara, J., Lund, J. R. \& Howitt, R. (2009). Hydroeconomic models: concepts, design, applications and future prospects. J. of Hydrol., 375, 334-350.

[8]. Brouwer, R. \& Hofkes, M. (2008). Integrated hydro-economic modelling: approaches, key issues and future research directions. Ecological Economics, 66, 16-22

[9]. Heinz, I., Pulido-Velasquez, M., Lund, J. \& Andreu, J. (2007). Hydro-economic modeling in river basin management: implications and applications for the European Water Framework Directive. Water Resour Manage, 21, 1103-1125.

[10]. Griffen, G. (1999). Watershed councils: an emerging form of public participation in natural resource management. Journal of the American Water Resources Association, 35, 505- 518.

[11]. Beck, M. B., Fath, B. D., Parker, A. K., Osidele, O. O., Cowie, G. M., Rasmussen, T. C., Patten, C., Norton, B. G., Steinmann, A., Borrett, S. R., Cox, D., Mayhew, M. C., Zeng, X. Q. \& Zeng, W. (2002). Developing a concept of adaptive community learning: case study of a rapidly urbanizing watershed. Integr. Assess., 3(4), 299-307.

[12]. Bagheri, A. (2006). Sustainable development: implementation in urban water systems. Dissertation, Lund University, Lund, Sweden

[13]. Madani, K. (2009). Climate Change Effects on High-Elevation Hydropower System in California. Ph.D. Dissertation, Department of Civil and Environmental Engineering, University of California, Davis

[14]. Singh, V. P. \& Frevert, D. K. eds. (2006). Watershed Models. CRC Press, Boca Raton, Florida.

[15]. Fieldman, A.D. (1979) Flood Hydrograph and Peak Flow Frequency Analysis. U.S. Army Corps of Engineers, Hydrologic Engineering Centre Technical Paper no. 62

[16]. Shawul, A. A., Alamirew, T., and Dinka, M. O.: Calibration and validation of SWAT model and estimation of water balance components of Shaya mountainous watershed, Southeastern Ethiopia, Hydrol. Earth Syst. Sci. Discuss., 10, 13955-13978, doi:10.5194/hessd-10-13955-2013, 2013.

[17]. Dendrou, S.A. 1982. Overview of Urban Stormwater Models, in Urban Stormwater Hydrology, D.F. Kibler (ed.), American Geophysical Union, Water Resources Monograph No. 7, Washington, DC, pp. 219-247.

[18]. Gan, T.Y., and S.J. Burges. 1990. An Assessment of a Conceptual Rainfall-Runoff Model's Ability to Represent the Dynamics of Small Hypothetical Catchments: 2. Hydrologic Responses for Normal and Extreme Rainfall. Water Resources Research 26 (7): 1605-1619.

[19]. Tohini, E., and J.R. Wallis. 1977. Using CLS for Daily or Longer Period Rainfall-Runoff Modeling. In Mathematical Models for Surface Water Hydrology, T.A. Cirinai, U. Maione, and J.R. Wallis (eds.), John Wiley \& Sons, New York, NY, pp. 149-168.

[20]. Larson, C.L. 1973. Hydrologic Effects of Modifying Small Watersheds -- Is Prediction by Hydrologic Modeling Possible? Transactions of the [ASAE 16: 560-564, 568

[21]. Edsel B. Daniel, Janey V. Camp, Eugene j. LeBoeuf, Jessica R. Penrod, James P. Dobbins and mark D. Abkowitz. Watershed modeling and its applications:a state of the art review. The open hydrology Journal, 2011,5,26-50

[22]. Ficklin, D. L., Luo, Y. and Zhang, M. (2013), Watershed modelling of hydrology and water quality in the Sacramento River watershed, California. Hydrol. Process., 27: 236-250. doi: 10.1002/hyp.9222

[23]. Franchini, M., and M. Pacciani. 1991. Comparative Analysis of Several Conceptual Rainfall-Runoff Models. Journal of Hydrology 122:161-219.

[24]. Woolhiser, D.A. and D.L. Brakensiek. 1982. Hydrologic Modeling of Small Watersheds. In Hydrologic Modeling of Small Watersheds, C.T. Haan, H.P. Johnson, and D.L. Brakensiek (eds.), American Society of Agricultural Engineers, Monograph No. 5, St. Joseph, MI, pp. 291-312.

[25]. James, L.D., and S.J. Burges. 1982a. Precipitation-Runoff Modeling: Future Directions. In Applied Modeling in Catchment Hydrology, V.P. Singh (ed.), Proceedings of the International Symposium on Rainfall-Runoff Modeling, Water Resources Publications, Littleton, CO, pp. 291-312.

[26]. Anderson, M.G. and T.P. Burt. 1985. Modelling Strategies. In Hydrological Forecasting, M.G. Anderson and T.P. Burt (eds.), John Wiley \& Sons, New York, NY, pp. 1-13. 